\title{
From Basel to Brussels: the FIP Basel Statements as the foundation of the EAHP Statements of Hospital Pharmacy
}

\author{
Lee Vermeulen
}

\section{Correspondence to}

Professor Lee Vermeulen, UW Health Center for Clinical Knowledge Management, Clinical Professor of Pharmacy, University of WisconsinMadison, 600 Highland Avenue, M/C 9475, Madison, Wisconsin 53792 USA; LC.Vermeulen@hosp.wisc.edu

Received 24 July 2014 Accepted 29 July 2014

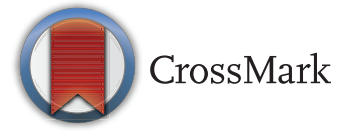

To cite: Vermeulen L. Eur J Hosp Pharm 2014:21: 262-263.

\section{ABSTRACT}

The International Pharmaceutical Federation (FIP) hosted the Global Conference on the Future of Hospital Pharmacy in 2008. Attended by 348 participants, representing 98 countries, the FIP Global Conference was a landmark event that produced what are now known as the FIP Basel Statements on the Future of Hospital Pharmacy. This paper provides a brief summary of the development and subsequent use of the Basel Statements on which the European Association of Hospital Pharmacists (EAHP) statements are based.

\section{INTRODUCTION}

In December 2005, the leaders of several hospital pharmacy organisations from around the world met at the Midyear Clinical Meeting of the American Society of Health-System Pharmacists (ASHP) to discuss the issues and concerns facing hospital pharmacists in their respective countries and regions. During that meeting, it was decided that a set of consensus statements articulating a shared global vision for the future of hospital pharmacy practice would benefit hospital pharmacists worldwide. Leaders from the Hospital Pharmacy Section of the International Pharmaceutical Federation (FIP) agreed to plan and host a consensus conference aimed at creating those statements.

Three years later, in August 2008, in Basel, Switzerland, FIP hosted the Global Conference on the Future of Hospital Pharmacy. Attended by 348 participants, representing 98 countries, the FIP Global Conference was a landmark event that produced what are now known as the FIP Basel Statements on the Future of Hospital Pharmacy. This paper provides a brief summary of the development and subsequent use of the Basel Statements on which the European Association of Hospital Pharmacists (EAHP) statements are based. ${ }^{1}$

\section{PREPARING FOR BASEL}

The FIP Global Conference on the Future of Hospital Pharmacy was an enormous undertaking that required leadership from many individuals. A Steering Committee provided guidance on the objectives and format of the Global Conference, and that group established several key guiding principles that framed the development of the Basel Statements.

The goal of the conference was to establish aspirational consensus statements. It was recognised that the development of hospital pharmacy practice is highly variable around the globe, with the level of advanced development generally linked to the resources available in each country and region. Our goal was not to create consensus statements exclusively for developed countries, or for those in developing regions, but rather to establish statements that are relevant across the continuum of development-establishing a vision for practice for the entire globe. We chose to develop statements that reflected a desired future for practice, recognising that achieving that desired future would be more difficult in some countries than in others.

The Steering Committee also decided very early on that it was essential to create evidence-based consensus statements-not simply statements that reflected the opinion of individuals, but instead focus on practice standards that were supported, to the greatest extent possible, by published evidence. The design of the conference was influenced significantly by this guiding principle.

Members of the Steering Committee were drawn from around the globe, and several European pharmacists and pharmaceutical scientists were recruited. Members included Jacqueline Surugue from France (who, with Henri Manasse, also served as the Honorary Co-Chair of the Global Conference), Arnold Vulto from The Netherlands (who also served as Chair of the Programming Subcommittee of the Conference), Stephen Curtis from the UK, Stefan Mühlebach from Switzerland and Dick Tromp from The Netherlands.

In preparation for the Global Conference, the FIP commissioned a survey to assess the current state of hospital pharmacy practice around the globe. ${ }^{2}$ Conducted at the University of WisconsinMadison in the USA, the survey drew on the experience other large national and regional survey efforts, particularly from previous surveys conducted by ASHP and EAHP, and provided an important picture of the scope and extent of practice development in various parts of the world. The survey results informed the development of consensus statements, and also established a baseline against which individual statements could be judged in terms of the degree to which they were reflective of current practice or aspirational as regards the potential future state of practice. The survey drew responses from 85 countries, including 29 European countries, and the results remain a rich source of information about the diversity of practice around the globe.

The development of the Basel Statements was also informed by exhaustive, systematic reviews of published literature on each of the themes selected 
by the Steering Committee. The Hospital Pharmacy Section recruited six faculty members, and tasked each with reviewing the literature on one specific aspect of hospital pharmacy practice and synthesising available evidence into primers on each topic. Each faculty member then used their primer as the basis for an on-line 'discussion' with FIP members, delegates preparing to attend the Global Conference and others interested in hospital pharmacy practice. Those discussions produced initial statements that formed the first draft of what would eventually become the Basel Statements. Faculty members represented all regions of the globe, and included David Cousins who provided leadership for the group focusing on statements regarding the monitoring of medication practice. ${ }^{1}$

The work done by these faculty members was overseen and coordinated by a dedicated Programming Subcommittee, led by Arnold Vulto. The conference was also supported by Satu Siiskonen, an outstanding hospital pharmacist from Erasmus University Hospital in Rotterdam, The Netherlands. The development of the Global Conference on the Future of Hospital Pharmacy, and the creation of the Basel Statements, benefited from a strong and consistent European influence, drawing on the expertise of hospital pharmacy leaders from throughout Europe.

\section{THE MOMENT}

Over the course of 2 days in Basel, hundreds of hospital pharmacists focused their considerable expertise on the creation of consensus statements. Drawing upon the work done in the months leading up to the Global Conference, and with the guidance of the Steering Committee members, Programming Subcommittee members and faculty, a set of draft consensus statements were finalised. On 31 August 2008, the assembled delegates represented at the conference voted on each draft statement. All were endorsed with very strong positive consensus, and the Basel Statements were born!

In his closing remarks at the conclusion of the FIP Global Conference on the Future of Hospital Pharmacy, Henri Manasse called on those who participated in the conference to recognise the event in its historical context, and challenged all hospital pharmacists to continue to pursue advances in hospital pharmacy practice. He described the closing of the conference as 'the moment' when we must all choose to recommit ourselves to reaching the aspirational goals set forth in the Basel Statements.

\section{CONTINUING THE WORK}

Since publishing the proceedings from the Global Conference on the Future of Hospital Pharmacy in March 2009, the FIP Hospital Pharmacy Section has done considerable work on implementing the Basel Statements. The Basel Statements have been translated into over 20 languages, including the six official languages of the United Nations (Arabic, Chinese, English, French, Russian and Spanish). ${ }^{3}$ Section members from every region of the world have completed projects on Basel Statement implementation. In the Western Pacific region, in particular, several projects have been completed and reports from those efforts have been published. ${ }^{4-6}$

As with all resources related to healthcare, work is needed to keep consensus statements current and reflective of contemporary practice. The need to revise and update the Basel Statements was considered almost immediately after they were first published. The FIP Hospital Pharmacy Section began making specific plans for a revision effort in late 2011, and that process began in 2013. The Hospital Pharmacy Section conducted another survey, collecting input from over 300 hospital pharmacists from around the globe on areas of the Basel Statements that require clarification and revision. While the main purpose of the revision has been to simplify the language used in many Statements, efforts have also gone into updating the Statements to reflect changes in practice standards that have taken place since 2008. Revisions to the Basel Statements will be completed in September 2014 at the FIP Congress in Bangkok, Thailand.

\section{REFLECTIONS ON THE EAHP STATEMENTS}

Through various regional efforts to implement aspects of the FIP Basel Statements, it has been made clear that the diversity of hospital pharmacy practice must be taken into account when choosing a direction for practice development. FIP has deliberately avoided putting the Basel Statements into any sort of priority order, to avoid making judgments about the relative importance of any one statement or group of statements. We have recognised that regional and national priorities must be considered when it is being decided which Statements should become the focus for improvement efforts, particularly when resources are scarce.

The development of a European vision for the future of hospital pharmacy has allowed that aim to be explicitly considered. The EAHP statements are a reflection of the unique capabilities of European hospital pharmacists, are influenced by the stated priorities of European healthcare systems, and are reflective of the challenges faced by European patients and other healthcare providers. While neither FIP nor EAHP have plans to harmonise the two sets of statements, the work done by EAHP will clearly influence the revisions being considered to the Basel Statements. Allowing the Basel Statements to serve as the starting point for the development of the EAHP statements increases the likelihood that there will be consistencies where there should similarities, and also ensures that the EAHP statements are a reflection of the specific needs of European hospital pharmacy practice.

It was my great privilege to be allowed to participate in the development of the EAHP Statements. On behalf of the FIP Hospital Pharmacy Section, I wish to congratulate the members and leaders of EAHP on the development of an important tool that will certainly guide practice in Europe for many years to come.

\section{Competing interests None.}

Provenance and peer review Commissioned; internally peer reviewed.

\section{REFERENCES}

1 International Pharmaceutical Federation. Proceedings of the Global Conference on the Future of Hospital Pharmacy. Am J Health Syst Pharm 2009;66(Suppl 3):S1-S80.

2 Doloresco F, Vermeulen LC. Global survey of hospital pharmacy practice. Am J Health Syst Pharm 2009;66(Suppl 3):S13-9.

3 FIP Global Conference on the Future of Hospital Pharmacy Successfully Completed. FIP web site for the Basel Statements. http://www.fip.org/baseltranslations? page $=$ globalconf (accessed 23 Jul 2014).

4 Penm J, Moles $\mathrm{R}$, Wang $\mathrm{H}$, et al. Factors affecting the implementation of clinical pharmacy services in China. Qual Health Res 2014;24:345-56.

5 Penm J, Chaar B, Dechun J, et al. Formulary systems and pharmacy and therapeutics committees in the Western Pacific Region: exploring two Basel Statements. Am J Health Syst Pharm 2013;70:967-79.

6 Penm J, Chaar B, Moles R. Validating a hospital medicines formulary survey in the Western Pacific Region--a global hospital pharmacy initiative based on the Basel Statements. Res Social Adm Pharm 2012;8:298-308. 